\title{
Complete Mitogenome of Endangered and Endemic Nicobar Treeshrew (Tupaia Nicobarica) and Comparison with Other Scandentians
}

\section{Shantanu Kundu}

Zoological Survey of India

Avas Pakrashi

Zoological Survey of India

Manokaran Kamalakannan

Zoological Survey of India

Devkant Singha

Zoological Survey of India

Kaomud Tyagi

Zoological Survey of India

Chinnadurai Venkatraman

Zoological Survey of India

Vikas Kumar ( $\nabla$ vikaszsi77@gmail.com )

Zoological Survey of India

\section{Research Article}

Keywords: Threatened mammals, Mitogenomics, Genomic features, Phylogeny

Posted Date: September 8th, 2021

DOl: https://doi.org/10.21203/rs.3.rs-880056/v1

License: (c) (i) This work is licensed under a Creative Commons Attribution 4.0 International License.

Read Full License

Version of Record: A version of this preprint was published at Scientific Reports on January 18th, 2022.

See the published version at https://doi.org/10.1038/s41598-022-04907-7. 


\section{Abstract}

The Nicobar treeshrew (Tupaia nicobarica) is an endangered smaller mammal endemic to the Nicobar Island of the Andaman Sea, India regarded as an alternative experimental animal model in biomedical research. The present study aimed to assemble the first mitochondrial genome of $T$. nicobarica to elucidate evolutionary relationship. The structure and variation of the novel mitochondrial genome were analyzed and compared with other Scandentians. The complete mitogenome (17,164 bp) encodes 37 genes, including 13 protein-coding genes (PCGs), 22 transfer RNA (tRNAs), two ribosomal RNA (rRNAs), and one control region (CR). Most of the genes were encoded on majority strand, except nad6 and eight tRNAs. The nonsynonymous/synonymous ratio in all PCGs indicates strong negative selection among all Tupaiidae species. The comparative study of CRs revealed the occurrence of tandem repeats (CGTACA) found in T. nicobarica. The phylogenetic analyses ( $\mathrm{ML}$ and BA) showed distinct clustering of $T$. nicobarica with high branch supports and depict a substantial divergence time (11.4 to 18.8 MYR) from the ancestor lineage of Tupaiidae. The 16S rRNA dataset corroborates the taxonomic rank of two subspecies of T. nicobarica from the Great and Little Nicobar Islands. The present study suggests the assembly of whole-genome to improve the understanding of evolutionary relationships of treeshrews and its implication in biomedical research.

\section{Introduction}

The biogeography of India is mainly classified into two categories, the mainland and groups of islands (Lakshadweep and Andaman-Nicobar). The Andaman and Nicobar (AN) archipelago was made up by submarine habitable mountains and constituted with 572 chain of islands located on the Bay of Bengal ${ }^{1}$. The AN archipelago was formed due to collision between the Indian Plate and Eurasian Plate which commenced about 50 million years ago and continues today ${ }^{2}$. Due to the unplugged landmass from the mainland of India and other diachronic makeup, this group of islands accommodates numerous unique elements of biodiversity ${ }^{3}$. Thus, these islands ecosystems were thought to be a trenchant biogeographic entity which can be evaluated to be a substantial model for evolutionary studies ${ }^{4,5}$. The study on faunal diversity of AN archipelago has been started since 19th century, considering the large size or charismatic vertebrate fauna, including mammals, birds, and herpetofauna ${ }^{6-8}$. Due to the remoteness and inaccessibility throughout the year, most of the regions of AN archipelago are sparsely explored. Remarkably, these oceanic islands also provide a suitable habitat for many smaller mammals like, treeshrews (order Scandentia), shrews (order Eulipotyphla), and rodents (order Rodentia) ${ }^{9}$.

The world treeshrew account for 23 species under four genera (Anathana, Dendrogale, Ptilocercus and Tupaia) of two families (Tupaiidae and Ptilocercidae) which are distributed in South Asia, Southeast Asia, and Southwest China ${ }^{10,11}$. The mainland of India is known by two species, the Madras treeshrew, Anathana ellioti and the Northern treeshrew, Tupaia belangeri. However, the Nicobar treeshrew, Tupaia nicobarica is endemic to the Nicobar Islands ${ }^{12}$. This species is categorized as 'Endangered' species in the IUCN Red List of Threatened Species and listed under 'Appendix II' in the Convention on International 
Trade in Endangered Species of Wild Fauna and Flora (CITES) ${ }^{13}$. On the basis of geographical distribution, two subspecies have been recognized, viz., T. nicobarica nicobarica from the Great Nicobar Island and T. nicobarica surda from the Little Nicobar Island ${ }^{14}$. Later on, these subspecies status has been endorsed by certain researchers and hinted further molecular investigation required to corroborate their taxonomic status ${ }^{12,15,16}$. Besides, the zoonotic disease has been originated or transmitted through different mammalian species and cause life threatening to human beings throughout the globe ${ }^{17,18}$. The treeshrews (order Scandentia) species is evidenced to be a significant model for various human disorders like, depression, myopia, hepatitis $B$ and $C$ virus infections, and hepatocellular carcinoma ${ }^{19,20}$. The molecular study and genome sequencing has been demonstrated the genetic basis of signaling pathways in nervous and immune systems of the Chinese treeshrew and evidenced as a potential model for biomedical research ${ }^{21}$.

The characterizations of complete mitogenomes are widely used genomics approaches for systematics studies and evolutionary research of wider group of taxa including mammals ${ }^{22-24}$. So far, the complete mitogenome of five species, Tupaia belangeri, Tupaia minor, Tupaia montana, Tupaia splendidula, and Tupaia tana were generated from different geographical regions. The molecular studies were previously aimed to infer the phylogenetic and evolutionary relationship, genetic structure, and possible gene flow of Scandentia across their range distribution in Southeast $A$ sia $^{25,26}$. In addition, the partial mitochondrial genes (12S rRNA, 16S rRNA, and Cytochrome b) were also utilized to elucidate the phylogenetic position and diversification of Tupaiidae species including T. nicobarica ${ }^{15,27,28}$. However, the in-depth genetic information and structural motifs of T. nicobarica mitogenome is still anonymous to the scientific communities. To fill the gap of knowledge, the present study aimed to determine the complete mitogenome of $T$. nicobarica from AN archipelago, India. The comparative analyses were confronted to check the structure and variation within the Tupaiidae mitogenomes. The phylogenetic analyses and divergence time were estimated to infer the evolutionary relationship of T. nicobarica comparing with other Tupaiidae species. Further, an additional dataset of mitochondrial 16S rRNA was also constructed to clarify the taxonomic rank of the extant subspecies of T. nicobarica in the Great and Little Nicobar Island.

\section{Materials And Methods}

\section{Sample collection and DNA extraction}

The museum sample of T. nicobarica is vouchered at the National Zoological Collections of Zoological Survey of India, which was collected from the Galathia, Campbell bay (06.51 N 93.50 E). No treeshrew specimen was killed; hence no prior permission was required in the present study. The tissue sample was aseptically collected from the archival specimen with ample attention and stored in $70 \%$ ethanol for downstream molecular investigation. The collection locality map was prepared by the online platform (https://noaa.maps.arcgis.com). The tissue sample was homogenized with $1 \mathrm{ml}$ buffer containing 0.32 M Sucrose, $1 \mathrm{mM}$ EDTA, and $10 \mathrm{mM}$ TrisHCl by the WiseTis HG-15 homogenizer. To remove the nuclei 
and cell debris, the working mixture was centrifuged at $700 \mathrm{~g}$ for $5 \mathrm{~min}$ at $4^{\circ} \mathrm{C}$. The supernatant was collected in $1.5 \mathrm{ml}$ centrifuge tube and centrifuged at $12,000 \mathrm{~g}$ for $10 \mathrm{~min}$ at $4^{\circ} \mathrm{C}$ to precipitate the mitochondrial pellet. The pellet was re-suspended in $500 \mu \mathrm{l}$ of buffer $(50 \mathrm{mM}$ TrisHCl, $25 \mathrm{mM}$ of EDTA, $150 \mathrm{mM} \mathrm{NaCl})$ and incubated at $56^{\circ} \mathrm{C}$ for $1-2 \mathrm{hr}$ along with $20 \mu \mathrm{l}$ of proteinase $\mathrm{K}(20 \mathrm{mg} / \mathrm{ml})$. The mitochondrial DNA was extracted by using QIAamp DNA Investigator Kit (QIAGEN Inc.) and the eluted volume was reduced to $100 \mu \mathrm{l}$ to increase the mtDNA concentration. The quality of the extracted mtDNA was checked through $1 \%$ agarose gel electrophoresis, and the concentration was quantified with a NANODROP 2000 spectrophotometer (Thermo Scientific).

\section{Sequencing, assembly and annotation}

The complete mitogenome sequence and assembly were carried out at PHIXGEN Pvt. Ltd. Gurugram, India (http://www.phixgen.com). The mitochondrial DNA (>100 ng) was used in Illumina TruSeq Nano DNA HT library preparation kit for library assembly (Illumina, Inc, USA). The mtDNA was fragmented by ultra-sonication and the A-tailed fragments were joined with the sequencing indexed adapters (Covaris M220, Covaris Inc., Woburn, MA, USA). The mtDNA fragments (450 bp) were selected through sample purification beads and enrich by polymerase chain reaction (PCR). The amplified PCR library was examined using Bioanalyzer 2100 (Agilent Technologies, Inc., Waldbronn, Germany) with high sensitivity DNA chips. Total >4 million raw reads were generated through Illumina NextSeq500 ( $150 \times 2$ chemistry) after obtaining the expected concentration $(650.25 \mathrm{pg} / \mu \mathrm{l})$ and mean peak size (465 bp) (Illumina, Inc, USA). To obtain the considerable sequencing depth (>75000X), the raw nucleotide reads were screened using cutadapt tool (http://code.google.com/p/cutadapt/) and trimmed with a cut-off of Phred quality scores of Q20. The high-quality reads were downsampled to 2 million using Seqtk

(https://github.com/lh3/seqtk) and assembled using NOVOPlasty v2.6.7 using default parameters ${ }^{29}$. The mitogenome of T. belangeri (accession no. NC_002521) was used as a reference seed sequence for assembly. The typical circular representation of the generated mitogenome of $T$. nicobarica was plotted by CGView Server (http://stoth ard.afns.ualbe rta.ca/cgview_server/) with default parameters ${ }^{\mathbf{3 0}}$. Further, the contig was subjected to confirmation by the MITOS v806 online webserver (http://mitos.bioinf.unileipzig.de). The direction and arrangements of PCGs, tRNAs, and rRNAs were confirmed through MITOS online server (http://mitos.bioinf.uni-leipzig.de) ${ }^{31}$. The start and stop codons of each PCG were assured through the Open Reading Frame Finder web tool (https://www.ncbi.nlm.nih.gov/orffinder/) on the basis of vertebrate mitochondrial genetic code and other publicly available reference sequences of Tupaiidae. The mitogenome was submitted to the GenBank database (Accession No. MW751815) using the NCBI Bankit submission tool.

\section{Dataset construction and comparative analysis}

On the basis of taxonomic classification, the mitogenomes of five Tupaiidae species were downloaded from GenBank and merged in the dataset for comparative analysis (Supplementary Table S1). The genome sizes and nucleotide compositions of all the studied species were calculated using MEGA $X^{32}$. To calculate the base composition skew, we utilized previously known formula: AT skew $=(A-T) /(A+T)$, 
$\mathrm{GC}$ skew $=(\mathrm{G}-\mathrm{C}) /(\mathrm{G}+\mathrm{C})^{33}$. The overlapping regions and intergenic spacers of $T$. nicobarica and other Tupaiidae species mitogenomes were calculated manually. The pairwise test of the synonymous (Ks) and non-synonymous (Ka) substitutions were calculated between T. nicobarica and other Tupaiidae species using DnaSPv6.0 ${ }^{34}$. The comparative analysis of relative synonymous codon usage (RSCU) and relative abundance of amino acids were also calculated using MEGA X. The secondary structures of tRNA genes were affirmed by tRNAscan-SE Search Server 2.0 (http://lowel ab.ucsc.edu/tRNAs can-SE/) and ARWEN 1.2 $2^{35,36}$. To speculate the putative domains and motif, the CR of T. nicobarica and other Tupaiidae species was screened from the database. The tandem repeats within the CR were predicted by the online Tandem Repeats Finder web tool (https://tandem.bu.edu/trf/trf.html) ${ }^{37}$.

\section{Phylogenetic analysis and divergence time estimation}

To assess the phylogenetic relationship, the dataset was constructed with the representatives of Scandentia, Dermoptera, and Primates based on the previous literature ${ }^{15}$. The 13 PCGs of 14 mitogenomes were aligned and concatenated using TranslatorX (with MAFFT algorithm with L-INS-i strategy and GBlocks parameters) and SequenceMatrix v1.7.8453738,39. The best fit model (GTR+l+G) was estimated by PartitionFinder 2 using lowest Bayesian information criterion (BIC) criterion ${ }^{40}$ and the maximum-likelihood (ML) tree was constructed using the IQ-Tree web server with 1000 bootstrap support ${ }^{41}$. The estimation of divergence times among Tupaiidae species were calculated by Bayesian relaxed clock method in BEAST v2.4.7 ${ }^{42}$. The GTR+ I+G substitution model, empirical base frequencies, and relaxed uncorrelated log-normal clock with the Yule speciation model was applied as Tree prior. A total of four fossil calibration points were applied in the phylogeny to constraint the analysis as described in the previous study ${ }^{15,43-45}$ : (i) node A, 18 million years ago (MYA) log-normal prior as the minimum age of Tupaia based on the fossil of T. miocenica, (ii) node B, 23 MYA log-normal prior as the minimum age for the split between Pygathrix and Hylobates, (iii) node C, a normal prior for the primate outgroups with a mean of 77.5 MYA, and (iv) node D, the total tree height is considered as a normal prior with a mean of 90 MYA for Scandentia, Dermoptera, and Primates. Two independent Markov chain Monte Carlo (MCMC) runs were performed for 1,000,000 generations with $25 \%$ burn-in and trees sampled every 2000 generations. To combine these runs, log files were imported into LogCombiner of the BEAST package. The adequate MCMC mixing and convergence were estimated using the effective sample size (ESS) values (>200 in all parameters) in Tracer v.1.7.146. To reconstruct the Bayesian (BA) phylogeny, TreeAnnotator was used which is included in the BEAST package. The consensus tree was further visualized by FigTree 1.4.4 with a divergence time of $95 \%$ higher probability density (HPD) ${ }^{47}$.

In addition, to check the taxonomic rank of two named subspecies, T. nicobarica nicobarica from the Great Nicobar Island and T. nicobarica surda from the Little Nicobar Island, an additional dataset of $16 \mathrm{~S}$ rRNA gene was constructed, including 18 database sequences of 12 Tupaiidae species known from South and Southeast Asian countries (Supplementary Table S2). The 16S rRNA sequence of the Sunda 
flying lemur, Galeopterus variegatus (Accession No. NC_004031) was used as an out-group in the second dataset.

\section{Results And Discussion}

\section{Mitogenome structure and organization}

The mitogenome (17,164 bp) of the endangered Nicobar treeshrew, T. nicobarica was determined in the present study (GenBank accession no. MW751815). The mitogenome contained 37 genes, comprising 13 PCGs, 22 tRNAs, 2 rRNAs, and a major non-coding CR. Among them, nine genes (nad6 and eight tRNAs) were placed on the negative strand, while the remaining 28 genes were placed on the positive strand (Table 1, Fig. 1). In the order Scandentia, the length of the Tupaiidae mitogenome varied from 16,183 bp (T. montana) to 17,164 bp (T. nicobarica). All Tupaiidae species showed the same gene arrangement as observed in typical vertebrate's mitogenome ${ }^{48}$. The nucleotide composition of the T. nicobarica mitogenome was A+T biased (58.3\%), as in all Tupaiidae species ranging from $58.3 \%$ (T. nicobarica) to $59.72 \%$ ( T. tana) (Table 2). The AT skew and GC skew were 0.11 and -0.30 in the mitogenome of $T$. nicobarica. The comparative analysis showed that the AT skew ranged from 0.08 ( $T$. minor and $T$. tana) to 0.11 ( $T$. nicobarica) and the GC skew from -0.28 (T. minor, T. montana, T. splendidula, T. tana) to -0.30 (T. nicobarica) (Table 2). A total of 14 overlapping regions with a total length of $87 \mathrm{bp}$ were identified in $T$. nicobarica mitogenome. The longest overlapping region (43 bp) was observed between the ATP synthase F0 subunit 8 (atp8) and ATP synthase F0 subunit 6 (atp6). Further, a total of 14 intergenic spacer regions with a total length of $68 \mathrm{bp}$ were observed in T. nicobarica mitogenome with the longest region (33 bp) between tRNA-Asparagine $(\operatorname{trnN})$ and tRNA-Cysteine $(\operatorname{trnC})$ (Supplementary Table S3).

\section{Protein-coding genes}

The total length of PCGs was $11,410 \mathrm{bp}$ in T. nicobarica, which represents $66.47 \%$ of the complete mitogenome. The nucleotide composition of the T. nicobarica PCGs was A+T biased (57.95\%), as in all Tupaiidae species ranging from $57.95 \%$ (T. nicobarica) to $59.61 \%$ (T. tana) (Table 2). The AT skew and GC skew were 0.09 and -0.36 in the PCGs of T. nicobarica (Table 2). Most of the PCGs of T. nicobarica initiated with an ATG start codon; however, the ATC initiation codon was found in the NADH dehydrogenase subunit 2 (nad2), ATT in NADH dehydrogenase subunit 3 (nad3), ATA in NADH dehydrogenase subunit 5 (nad5). The TAG termination codon was used by six PCGs, TAA by four PCGs, AGA by Cytochrome oxidase subunit 1 (cox1), AGG by NADH dehydrogenase subunit 6 (nad6), and incomplete T(AA) by NADH dehydrogenase subunit 4 (nad4) respectively. The comparative study revealed that, most of the PCGs in other Tupaiidae species were initiated by ATG start codon and terminated by TAA stop codon (Supplementary Table S4). The analysis of mitogenome for detecting positive selection of PCGs assists to understand the influences of natural selection in evolution and protein function ${ }^{49,50}$. The comparison of synonymous (Ks) and nonsynonymous (Ka) substitution rates in PCGs, witnessed for Darwinian selection and adaptive molecular evolution ${ }^{\mathbf{5 1}, \mathbf{5 2}}$. It is reported that, for positive selection $\mathrm{Ka} / \mathrm{Ks}>1$, for neutrality $\mathrm{Ka} / \mathrm{Ks}=1$, and for negative selection $\mathrm{Ka} / \mathrm{Ks}<1^{53}$. This 
approach has the benefit to reveal the natural selection acting on PCGs. Thus, to investigate the evolutionary rates between homologous gene pairs, $\mathrm{Ka} / \mathrm{Ks}$ substitutions were calculated and compared with six Tupaiidae species. The average Ka/Ks values of 13 PCGs varied from 0.006 (cox1) to 0.153 (atp8) and resulted in the following order:

cox $1<\operatorname{cox} 3<\operatorname{cox} 2<$ atp $6<$ nad3<cytb<nad1<nad4<nad6<nad4|<nad5<nad2<atp8 (Supplementary Table S5, Supplementary Fig. S1). Most of the PCGs show Ka/Ks values of $<1$, which indicated a strong negative selection among the studied Tupaiidae species, that reflects natural selection works against deleterious mutations with negative selective coefficients as highlighted general patterns in other vertebrates. The comparative RSCU analysis indicated a significant fall in the frequency of GCG codon in Alanine (Ala) was observed in T. nicobarica, T. montana, T. minor, T. splendidula, and T. tana, except in T. belangeri with CCG in Proline (Pro) (Supplementary Fig. S2).

\section{Ribosomal RNA and transfer RNA genes}

The total length of two rRNA genes of $T$. nicobarica was 2,519 bp, compared to a range from 2,508 bp ( $T$. montana) to 2,520 bp ( $T$. belangeri) among other Tupaiidae species in the present dataset. The AT content within rRNA genes was $58.56 \%$, while the AT and GC skew were 0.22 and -0.09 respectively observed in T. nicobarica rRNAs (Table 2). A total of 22 tRNAs were found in the T. nicobarica mitogenome with a total length of 1,497 bp. In other Tupaiidae species, the length of tRNAs varied from $1,493 \mathrm{bp}$ ( $T$. minor) to $1,564 \mathrm{bp}$ ( $T$. belangeri). The AT content within tRNA genes was $60.86 \%$, while the AT and GC skew were 0.11 and -0.12 , respectively observed in T. nicobarica tRNAs (Table 2). Most of the tRNA genes were predicted to be folded into classical cloverleaf structures, except trnS1 (without DHU stem and loop) and trnK (without DHU loop) (Supplementary Fig. S3). The conventional pairings ( $A=T$ and $\mathrm{G} \equiv \mathrm{C}$ ) were observed in most of the tRNAs bases ${ }^{54}$; however, wobble base pairing was observed in the stem of 14 tRNAs (trnA, trnN, trnQ, trnE, trnC, trnG, trnL1, trnK, trnL2, trnP, trnS2, trnT, trnY, and trnW) (Supplementary Fig. S3). The wobble base pairing is a key feature of RNA structure and often substitutes the conventional base pairs due to thermodynamic stability. These characteristics play crucial functional roles in a wide range of phenomena ${ }^{55}$. Thus, the comparisons of tRNAs secondary structures are crucial for inferring the structural and functional features of the mitogenomes ${ }^{56}$.

\section{Control regions}

The CR of T. nicobarica was typically distributed with three functional domains: extended termination associated sequences (ETAS), central domain (CD), and the conserved sequence block (CSB), as observed in other mammalian mitochondrial $\mathrm{CRs}^{25,57}$. Although, the ETAS and CSB domains contain varying numbers of tandem repeats, the $C D$ domain consists with highly conserved sequences. Hence, the pattern of $\mathrm{CR}$ was varied among different mammals, including Tupaiidae (Scandentia). The total length of $T$. nicobarica CR was $1,757 \mathrm{bp}$, compared to a range of $778 \mathrm{bp}$ ( $T$. splendidula) to 1,757 bp ( $T$. nicobarica) in the present dataset. In the T. nicobarica CR, the AT and GC skew was 0.12 and -0.30 (Table 2). The $\mathrm{CR}$ is also involved in the initiation of replication and is positioned between trnP and trnF for most of the Tupaiidae including T. nicobarica. The ETAS domain was divided into two regions: ETAS1 (60 bp) 
and ETAS2 (67 bp), while the CSB domain was further divided into three regions: CSB1 (25 bp), CSB2 (17 bp), and CSB3 (18 bp). After CSB3, a six base pair (CGTACA) tandem repeats were found 60.3 times in $T$. nicobarica, while eight base pair (CACACATA) were found 23.8 times in T. belangeri (Fig. 2). Due to the short nucleotide length, no tandem repeats were found in other Tupaiidae species CRs. The structural features of CR play an important function in influencing transcription and replication in the mitochondrial genome ${ }^{58,59}$. The present study evaluated the genetic features of CR among the studied Tupaiidae species mitogenomes including T. nicobarica that will be helpful to speculate the evolutionary pattern of this group.

\section{Phylogenetic relationship}

The phylogenetic position of Scandentia is repetitively examined within the eutherian tree. The treeshrews are widely considered as living fossils due to their approximating ancestral lineages with primates ${ }^{60}$. Based on the anatomical evidence, Primates, Chiroptera, Dermoptera, and Scandentia were hypothetically within the superordinal clade Archonta without considering the paleontological or molecular evidence $^{61,62}$. Later on, the phylogenetic position of Scandentia has been studied based on the complete mitochondrial DNA sequences of wider group of taxa and corroborated a closer relationship with Lagomorpha $24,25,63$. Further, multiple loci of mitochondrial genes has been assessed to check the phylogeny of treeshrews and diversification and the timescale of diversification in Southeast Asia ${ }^{15,27,64,65}$. The present ML and BA phylogeny clearly discriminate T. nicobarica from other congeners by complete mitochondrial genome and congruent with earlier evolutionary hypotheses (Fig. 3, Supplementary Fig. S4). Further, using four calibration points from earlier studies, the present mitogenome-based dating analysis indicates that, the Tupaiidae species (Scandentia) were diverged from Primates and Dermoptera during the Cretaceous period (90 to 104.9 MYA). The studied Tupaiidae species, including T. nicobarica were diverged during the Pliocene to Miocene epoch (2.8 to 20.8 MYR). The endemic Nicobar treeshrew, T. nicobarica was diverged from the common ancestor lineages of other Tupaiidae species during the Miocene epoch (11.4 to 18.8 MYR) (Fig. 3). Further, based on 16S rRNA genes (1667 bp), we evaluated the status of two known subspecies of T. nicobarica from the Great and Little Nicobar Islands. The T. nicobarica nicobarica and T. nicobarica surda showed cohesive clustering in the BA tree as compared with other species (Fig. 4). Both the subspecies depicted 11 variable sites and maintained less genetic distance $(0.7 \%)$ with each other. The $16 \mathrm{~S}$ rRNA based topology showed a sister relationship of $T$. nicobarica with $T$. javanica, distributed in Sumatra and Java.

\section{Biogeographic connection and conservation implication}

Due to the volcanic eruption in Miocene-Pliocene and subsequent irregular land bridges during the Pleistocene, several small to large island ecosystems were built up in South and Southeast Asia. The South and Southeast Asian mainland and islands have been formed by the convergence of three major crustal units of the earth, the Eurasian, Indo-Australian, and Pacific plates ${ }^{2}$. The tectonic drifts allowed multiple possibilities for animal dispersal and thus colonize many family members into the same or distant geographical distribution. Due to the adjacent biogeography realms, the biological connections 
between the Indian mainland and Southeast Asia were well documented ${ }^{66}$. However, the faunal diversity of the AN archipelago and their biotic networks is still anonymous in spectacular aspects. The bathymetric study evidenced that the well-developed seamounts have been detected on the Andaman seafloor, which extended up to Sumatra and Java Islands ${ }^{67,68}$. Considering the skeletal variation, the treeshrew species showed intraspecific variation depending upon their distribution in mainland and island ecosystems ${ }^{69}$. A recent molecular study also elucidates the biogeographic connection of smaller mammals in the AN archipelago with the Indo-Malayan and Sundaic realms ${ }^{3}$. The present mitogenome based phylogeny also manifested the close relationship of T. nicobarica with $T$. minor, T. $\operatorname{tana}, T$. splendidula, and T. montana (distributed in Thailand, Peninsular and East Malaysia, Brunei Darussalam, Sumatra, and Indonesia) as compared with the widespread species $T$. belangeri known from South and Southeast Asia. Further, the single gene (16S rRNA) based phylogeny showed sister relationship of $T$. nicobarica with T. javanica (distributed in Sumatra and Java) as compared with other congeners.

Considering the conservation implication, the previous studies reported that, this arboreal mammal species confronted several threats due to the forest loss and fragmentation, and ongoing road construction from Galathia to Indira Point at the Great Nicobar Island ${ }^{70}$. Although the species is listed on the IUCN with decreasing population trend, it has not yet listed in the Indian wildlife (Protection) Act, 1972. Other than a single ecology and behavior study and a nest record, no ample assessment has been approached so far ${ }^{12,71}$.

Besides, the treeshrews species were considered as a significant model for studying hepatitis and influenza H1N1 viral infections ${ }^{19,20}$. A recent study characterized the genome sequence to demonstrate the genetic basis of signaling pathways in nervous and immune systems of the Chinese treeshrew and evidenced as a potential model for biomedical research ${ }^{21}$. We propose the whole genome sequencing of T. nicobarica is essential for improving our ability to predict the signaling pathways linked with many pathogenic microorganisms as well as able to develop potential mitigations programs in advance. As the population of this treeshrew is confined to the insular habitats in the Nicobar Islands, we propose an integrated approach with taxonomy, ecology, and further molecular studies to save this endemic species before it reaches to the brink of extinction.

\section{Declarations}

\section{Acknowledgments}

The authors thankful to the Director of Zoological Survey of India (ZSI), Ministry of Environment, Forests and Climate Change (MoEF\&CC), Govt. of India. This work was financially supported by the Ministry of Environment, Forest and Climate Change (MoEF\&CC), Zoological Survey of India (ZSI) in-house project, 'National Faunal Genome Resources (NFGR)' to V.K. The first author (S.K) acknowledges a fellowship grant received from the Council of Scientific and Industrial Research (CSIR) Senior Research Associateship (Scientists' Pool Scheme) Pool No. 9072-A. 


\section{Funding}

Ministry of Environment, Forest and Climate Change (MoEF\&CC), Zoological Survey of India (ZSI) inhouse project, 'National Faunal Genome Resources (NFGR).

\section{Declaration of Interest Statement}

The authors declare that they have no competing interests.

\section{Author Contributions}

Conceptualization: S.K., V.K.; Data curation: S.K. M.K.; Formal analysis: S.K., A.P., D.S.; Funding acquisition: C.V., V.K.; Investigation: S.K., K.T.; Methodology: S.K. M.K., A.P., D.S.; Project administration: C.V., V.K.; Resources: C.V., V.K.; Software: S.K. K.T., A.P.; Supervision: C.V., V.K.; Validation: S.K., M.K.; Visualization: S.K., M.K., V.K.; Writing - original draft: S.K., M.K., A.P.; Writing - review \& editing: S.K., M.K., V.K.

\section{Data Availability}

The following information was supplied regarding the accessibility of DNA sequences: The generated complete mitochondrial genome sequences of Tupaia nicobarica are deposited in GenBank of NCBI under accession number MW751815.

\section{ORCID IDs}

Shantanu Kundu: https://orcid.org/0000-0002-5488-4433

Avas Pakrashi: https://orcid.org/0000-0001-5876-0203

Manokaran Kamalakannan: https://orcid.org/0000-0003-4440-0041

Devkant Singha: https://orcid.org/0000-0002-0670-520X

Kaomud Tyagi: https://orcid.org/0000-0003-1064-9826

Chinnadurai Venkatraman: https://orcid.org/0000-0002-9593-5727

Vikas Kumar: http://orcid.org/0000-0002-0215-0120

\section{References}

1. Yahya, H. S. A. \& Zarri, A. A. Status, ecology and behaviour of Narcondam Hornbill (Aceros narcondami) in Narcondam Island, Andaman and Nicobar Islands, India. J. Bom. Nat. His. Soc. 99, 434-445 (2002). 
2. Datta-Roy, A. \& Karanth K. P. The Out-of-India hypothesis: What do molecules suggest? J. Biosci. 34, 687-697 (2009).

3. Kamalakannan, M., Sivaperuman, C., Kundu, S. et al. Discovery of a new mammal species (Soricidae: Eulipotyphla) from Narcondam volcanic island, India. Sci Rep 11, 9416 (2021). https://doi.org/10.1038/s41598-021-88859-4

4. Matthews, T. J., Rigal, F., Triantis, K. A. \& Whittaker, R. J. A global model of island species-area relationships. Proc. Natl. Acad. Sci. USA 116, 12337-12342 (2019).

5. Upham, N. S., Esselstyn, J. A. \& Jetz, W. Ecological causes of uneven diversification and richness in the mammal tree of life. BioRxiv. https://doi.org/10.1101/504803 (2019).

6. Davidar, P. Yoganand, K. \& Ganesh, T. Distribution of forest birds in the Andaman Islands: importance of key habitats. J. Biogeogr. 28, 663-671 (2001).

7. Harikrishnan, S. et al. Macroecology of Terrestrial Herpetofauna in Andaman \& Nicobar Archipelago. Wildlife Institute of India, Uttarakhand, India. 1-49 (2014).

8. Kamalakannan, M. \& Venkatraman, C. A Checklist of Mammals of India (Zoological Survey of India, 2017).

9. Menon, V. Indian Mammals-A Field Guide 528 (Hachette Book Publishing India Pvt Limited, Gurgaon, 2014).

10. Burgin, C. J., Colella, J. P., Kahn, P. L. \& Upham, N. S. How many species of mammals are there? J. Mamm. 99, 1-14 (2018).

11. Wilson, D. E. \& Mittermeier, R. A. Handbook of the Mammals of the World, Vol 8: Insectivores, Sloths and Colugos 709 (Lynx Edicions, 2018).

12. Oommen, M. A. \& Shanker, K. Ecology and behaviuour of endemic treeshrew Tupaia nicobarica Zelebor 1869 on Great Nicobar Island, India. J. Bom. Nat. Hist. Soc. 105, 55-63 (2008).

13. IUCN. The IUCN Red List of Threatened Species, Version 2020-3 (IUCN, 2020).

14. Miller, G. S. Mammals of the Andaman and Nicobar Islands. Proc. U.S Nat. Mus. 24, 751-795 (1902).

15. Roberts, T. E., Lanier, H.C., Sargis, E. J. \& Olson, L.E. Molecular phylogeny of treeshrews (Mammalia: Scandentia) and the timescale of diversification in Southeast Asia. Mol. Phylogenet. Evol. 60, 358372 (2011).

16. Oommen, M. A. Treeshrews. In Mammals of South Asia (eds Johnsingh, A. J. T. \& Manjrekar, N.). University Press, Hyderabad 52-67 (2013).

17. Damas, J. et al. Broad host range of SARS-CoV-2 predicted by comparative and structural analysis of ACE2 in vertebrates. Proc. Natl. Acad. Sci. USA 117, 22311-22322 (2020).

18. Wardeh, M., Baylis, M. \& Blagrove, M. S. C. Predicting mammalian hosts in which novel coronaviruses can be generated. Nat. Commun. 12, 780. (2021)

19. Cao, J., Yang, E-B, Su, J-J., Li, Y. \& Chow, P. The tree shrews: adjuncts and alternatives to primates as models for biomedical research. J. Med. Primato/32, 123-130 (2003). 
20. Yang, Z. F. et al. The tree shrew provides a useful alternative model for the study of influenza H1N1 virus. Virol. J. 10, 111 (2013)

21. Yu, F. et al. Genome of the Chinese tree shrew. Nat Commun 4, 1426 (2013).

22. Pacheco, M. A. et al. Escalante, Evolution of modern birds revealed by mitogenomics: timing the radiation and origin of major orders. Mol. Biol. Evol. 28, 1927-42 (2011).

23. Finstermeier, K. et al. A Mitogenomic Phylogeny of Living Primates. PLoS ONE 8, e69504 (2013).

24. Arnason, U. et. al. Mammalian mitogenomic relationships and the root of the eutherian tree. Proc. Natl. Acad. Sci. USA 99, 8151-6 (2020).

25. Schmitz, J. Ohme, M. \& Zischler, H. The complete mitochondrial genome of Tupaia belangeri and the phylogenetic affiliation of scandentia to other eutherian orders. Mol. Biol. Evol. 17, 1334-43 (2000).

26. Parker, D. et al. Little genetic structure in a Bornean endemic small mammal across a steep ecological gradient. Mol. Ecol. 29, 4074-4090 (2020).

27. Olson, L. E., Sargis, E. J. \& Martin, R. D. Intraordinal phylogenetics of treeshrews (Mammalia: Scandentia) based on evidence from the mitochondrial 12S rRNA gene. Mol. Phylogenet. Evol. 35, 656-73 (2005).

28. Kundu, S. et al. Molecular investigation of non-volant endemic mammals through mitochondrial cytochrome b gene from Andaman and Nicobar archipelago. Mitochondrial DNA B 5, 1447-1452 (2020).

29. Dierckxsens, N., Mardulyn, P. \& Smits, G. NOVOPlasty: de novo assembly of organelle genomes from whole genome data. Nucleic Acids Res. 45, e18 (2017).

30. Grant, J. R. \& Stothard, P. The CGView Server: a comparative genomics tool for circular genomes. Nucleic Acids Res. 36, W181-184 (2008).

31. Bernt, M. et al. MITOS: Improved de novo Metazoan Mitochondrial Genome Annotation. Mol. Phylogenetic. Evolution 69, 313-319 (2013).

32. Kumar, S., Stecher, G., Li, M., Knyaz, C. \& Tamura, K. MEGA X: Molecular evolutionary genetics analysis across computing platforms. Mol. Biol. Evol. 35, 1547-1549 (2018).

33. Perna, N. T. \& Kocher, T. D. Patterns of nucleotide composition at fourfold degenerate sites of animal mitochondrial genomes. J. Mol. Evol. 41, 353-359 (1995).

34. Rozas, J. et al. DnaSP 6: DNA sequence polymorphism analysis of large data sets. Mol. Biol. Evol. 34, 3299-3302 (2017).

35. Laslett, D. \& Canbäck, B. ARWEN, a program to detect tRNA genes in metazoan mitochondrial nucleotide sequences. Bioinformatics 24, 172-175 (2008).

36. Lowe, T. M. \& Chan, P. P. tRNAscan-SE On-line: Search and Contextual Analysis of Transfer RNAGenes. Nucleic Acids Res. 44, W54-57 (2016).

37. Benson, G. Tandem repeats finder: A program to analyze DNA sequences, Nucleic Acids Res. 27, 573580 (1999). 
38. Abascal F., Zardoya, R. \& Telford, M. J. TranslatorX: multiple alignment of nucleotide sequences guided by amino acid translations. Nucleic Acids Res. 38, W7-13 (2010).

39. Vaidya, G., Lohman. D. J. \& Meier, R. J. SequenceMatrix: concatenation sofware for the fast assembly of multigene datasets with character set and codon information, Cladistics 27, 171-180 (2010).

40. Lanfear, R., Frandsen. P. B., Wright, A. M. Senfeld, T. \& Calcott, B. PartitionFinder 2: New Methods for Selecting Partitioned Models of Evolution for Molecular and Morphological Phylogenetic Analyses. Mol. Biol. Evol. 34, 772-773 (2016).

41. Trifinopoulos, J., Nguyen, L.-T., von Haeseler, A. \& Minh, B. Q. W-IQ-TREE: A fast online phylogenetic tool for maximum likelihood analysis. Nucleic Acids Res. 44, W232-W235 (2016).

42. Bouckaert, R. et al. BEAST2: a software platform for Bayesian evolutionary analysis. PLoS Comput Biol 10, e1003537 (2014).

43. Mein, P. \& Ginsburg, L. Les mammifères du gisement miocène inférieur de Li Mae Long, Thaïlande: systématique, biostratigraphie et paléoenvironnement. Geodiversitas 19, 783-844 (1997).

44. Eizirik, E., Murphy, W. J., Springer, M. S. \& O’Brien, S. J. Molecular phylogeny and dating of early primate divergences. In Anthropoid origins (eds Ross, C. F \& Kay, R. F.). New visions. Kluwer Academic/Plenum Publishers, New York (2004).

45. Hedges, S.B., Dudley, J., \& Kumar, S. TimeTree: A public knowledge-base of divergence times among organisms. Bioinformatics 22, 2971-2972 (2006).

46. Rambaut, A., Drummond, A. J., Xie, D., Baele G. \& Suchard, M.A. Posterior summarisation in Bayesian phylogenetics using Tracer 1.7. Syst. Biol. 67, 901-904 (2018).

47. Rambaut, A. FigTree. Version 1.4.4 Institute of Evolutionary Biology, University of Edinburgh, Edinburgh (2014).

48. Anderson, S. et al. Complete sequence of bovine mitochondrial DNA conserved features of the mammalian mitochondrial genome. J. Mol. Evol. 156, 683-717 (1982).

49. Hirsh, A. E. \& Fraser. H. B. Protein dispensability and rate of evolution. Nature 411, 1046-1049 (2001).

50. Bloom, J. D., Labthavikul, S. T. \& Otey, C. R. Protein stability promotes evolvability. Proc. Natl. Acad. Sci. USA 103, 5869-5874 (2006).

51. Yang, Z. \& Bielawski, J. P. Statistical methods for detecting molecular adaptation. Trends Ecol. Evol. $15,496-503$ (2000).

52. Yang, Z. H. \& Nielsen, R. Estimating synonymous and nonsynonymous substitution rates under realistic evolutionary models. Mol. Biol. Evol. 17, 32-43 (2000).

53. Nei, M. \& Kumar, S. Molecular Evolution and Phylogenetics. Oxford University Press, New York (2000).

54. Varani, G. \& McClain, W. H. The G-U wobble base pair: A fundamental building block of RNA structure crucial to RNA function in diverse biological systems. EMBO Reports 1, 18-23 (2000). 
55. Crick, F. H. C. Codon-anticodon pairing: The wobble hypothesis. J. Mol. Biol. 19, 548-555 (1966).

56. Takashi, P. S., Miya, M. \& Mabuchi, K. Structure and variation of the mitochondrial genome of fishes. BMC Genomics 17, 719 (2016).

57. Sbisa, E., Tanzariello, F., Reyes, A., Pesole, G. \& Saccone, C. Mammalian mitochondrial D-loop region structural analysis: identification of new conserved sequences and their functional and evolutionary implications. Gene 205, 125-140 (1997).

58. Taanman, J. W. The mitochondrial genome: structure, transcription, translation and replication. Biochim Biophys Acta 1410, 103-23 (1999).

59. Shao, R., Barker, S. C., Mitani, H., Aoki, Y. \& Fukunaga, M. Evolution of duplicate control regions in the mitochondrial genomes of Metazoa: a case study with Australasian Ixodes ticks. Mol. Biol. Evol. 22, 620-9 (2005).

60. Li, Q. \& Ni, X. An early Oligocene fossil demonstrates treeshrews are slowly evolving "living fossils". Sci Rep 6, 18627 (2016).

61. Novacek, M. J. Mammalian phylogeny: shaking the tree, Nature 356, 121-125 (1992).

62. McKenna, M. C \& Bell, S. K. Classification of Mammals Above the Species Level. Columbia University Press, New York (1997).

63. Xu, L., Chen, S. Y. \& Nie, W. H. Evaluating the phylogenetic position of Chinese tree shrew (Tupaia belangeri chinensis) based on complete mitochondrial genome: implication for using tree shrew as an alternative experimental animal to primates in biomedical research. J. Genet Genomics 39, 131-7 (2012).

64. Roberts, T. E., Sargis, E. J. \& Olson, L. E. Networks, trees, and treeshrews: Assessing support and identifying conflict with multiple loci and a problematic root. Syst Bio/ 58, 257-270 (2009).

65. Zhou, X., Sun, F., Xu, S., Yang, G. \& Li, M. The position of tree shrews in the mammalian tree: Comparing multi-gene analyses with phylogenomic results leaves monophyly of Euarchonta doubtful. Integr. Zool. 10, 186-98 (2015).

66. Garg, S. \& Biju, S. D. New microhylid frog genus from Peninsular India with Southeast Asian affinity suggests multiple Cenozoic biotic exchanges between India and Eurasia. Sci. Rep. 9, 1906 (2019).

67. Rodolfo, K. S. Bathymetry and marine geology of the Andaman basin, and tectonic implications for Southeast Asia. Geol. Soc. Am. Bull. 80, 1203-1230 (1969).

68. Tripathi, S. K. et al. Morphology of submarine volcanic seamounts from inner volcanic arc of Andaman Sea. Indian J. Geosci. 71, 451-470 (2017).

69. Sargis, E. J., Woodman, N., Morningstar, N. C., Bell, T. N. \& Olson, L.E. Skeletal variation and taxonomic boundaries among mainland and island populations of the common treeshrew (Mammalia: Scandentia: Tupaiidae). Biol. J. Linn. Soc. 120, 286-312 (2017).

70. Molur, S. et al. Status of Non-volant Small Mammals: Conservation Assessment and Management Plan (CAMP) Workshop Report 618 (Zoo Outreach Organisation, 2005). 
71. Kamalakannan, M., Gokulakrishnan, G., Venkatraman, C., Sivaperuman, C. \& Chandra, K. First record of a Nicobar treeshrew nest in a fallen palm tree. Mammalia 85, 159-160 (2021).

\section{Tables}

Table 1: List of annotated mitochondrial genes of Tupaia nicobarica. 


\begin{tabular}{|c|c|c|c|c|c|c|c|}
\hline Gene & Direction & Location & $\begin{array}{l}\text { Size } \\
\text { (bp) }\end{array}$ & $\begin{array}{l}\text { Anti- } \\
\text { codon }\end{array}$ & $\begin{array}{l}\text { Start } \\
\text { codon }\end{array}$ & $\begin{array}{l}\text { Stop } \\
\text { codon }\end{array}$ & $\begin{array}{l}\text { Intergenic } \\
\text { Nucleotides }\end{array}$ \\
\hline $\operatorname{trn} F$ & + & $1-66$ & 66 & GAA & - & - & -1 \\
\hline$r r n S$ & + & $66-1013$ & 948 & - & - & - & 4 \\
\hline $\operatorname{trn} V$ & + & $\begin{array}{l}1018- \\
1084\end{array}$ & 67 & TAC & - & - & 0 \\
\hline$r r n L$ & + & $\begin{array}{l}1085- \\
2655\end{array}$ & 1571 & - & - & - & 0 \\
\hline $\operatorname{trn} L 2$ & + & $\begin{array}{l}2656- \\
2730\end{array}$ & 75 & TAA & - & - & 2 \\
\hline nad1 & + & $\begin{array}{l}2733- \\
3689\end{array}$ & 957 & - & ATG & TAG & -2 \\
\hline trnl & + & $\begin{array}{l}3688- \\
3756\end{array}$ & 69 & GAT & - & - & -3 \\
\hline $\operatorname{trn} Q$ & - & $\begin{array}{l}3754- \\
3824\end{array}$ & 71 & TTG & - & - & -1 \\
\hline $\operatorname{trn} M$ & + & $\begin{array}{l}3824- \\
3892\end{array}$ & 69 & CAT & - & - & 0 \\
\hline nad2 & + & $\begin{array}{l}3893- \\
4936\end{array}$ & 1044 & - & ATC & TAG & -2 \\
\hline $\operatorname{trn} W$ & + & $\begin{array}{l}4935- \\
5001\end{array}$ & 67 & TCA & - & - & 4 \\
\hline $\operatorname{trn} A$ & - & $\begin{array}{l}5006- \\
5075\end{array}$ & 70 & TGC & - & - & 1 \\
\hline $\operatorname{trn} N$ & - & $\begin{array}{l}5077- \\
5149\end{array}$ & 73 & GTT & - & - & 33 \\
\hline $\operatorname{trn} C$ & - & $\begin{array}{l}5183- \\
5249\end{array}$ & 67 & GCA & - & - & 0 \\
\hline $\operatorname{trn} Y$ & - & $\begin{array}{l}5250- \\
5315\end{array}$ & 66 & GTA & - & - & 1 \\
\hline $\cos 1$ & + & $\begin{array}{l}5317- \\
6864\end{array}$ & 1548 & - & ATG & $A G A$ & -5 \\
\hline $\operatorname{trnS2}$ & - & $\begin{array}{l}6860- \\
6928\end{array}$ & 69 & TGA & - & - & 4 \\
\hline $\operatorname{trn} D$ & + & $\begin{array}{l}6933- \\
7001\end{array}$ & 69 & GTC & - & - & 0 \\
\hline $\cos 2$ & + & $\begin{array}{l}7002- \\
7685\end{array}$ & 684 & - & ATG & TAG & 1 \\
\hline
\end{tabular}




\begin{tabular}{|c|c|c|c|c|c|c|c|}
\hline trnK & + & $\begin{array}{l}7687- \\
7750\end{array}$ & 64 & TTT & - & - & 2 \\
\hline atp8 & + & $\begin{array}{l}7753- \\
7956\end{array}$ & 204 & - & ATG & TAA & -43 \\
\hline atp6 & + & $\begin{array}{l}7914- \\
8594\end{array}$ & 681 & - & ATG & TAA & -1 \\
\hline $\operatorname{cox} 3$ & + & $\begin{array}{l}8594- \\
9379\end{array}$ & 786 & - & ATG & TAA & -1 \\
\hline $\operatorname{trn} G$ & + & $\begin{array}{l}9379- \\
9445\end{array}$ & 67 & TCC & - & - & 9 \\
\hline nad3 & + & $\begin{array}{l}9455- \\
9802\end{array}$ & 348 & - & ATT & TAG & -10 \\
\hline $\operatorname{trn} R$ & + & $\begin{array}{l}9793- \\
9858\end{array}$ & 66 & TCG & - & - & 1 \\
\hline nad4l & + & $\begin{array}{l}9860- \\
10156\end{array}$ & 297 & - & ATG & TAA & -7 \\
\hline nad4 & + & $\begin{array}{l}10150- \\
11527\end{array}$ & 1378 & - & ATG & $\mathrm{T}(\mathrm{AA})$ & 0 \\
\hline $\operatorname{trnH}$ & + & $\begin{array}{l}11528- \\
11596\end{array}$ & 69 & GTG & - & - & 0 \\
\hline trnS1 & + & $\begin{array}{l}11597- \\
11655\end{array}$ & 59 & GCT & - & - & -1 \\
\hline $\operatorname{trn} L 1$ & + & $\begin{array}{l}11655- \\
11724\end{array}$ & 70 & TAG & - & - & -9 \\
\hline nad5 & + & $\begin{array}{l}11716- \\
13536\end{array}$ & 1821 & - & ATA & TAG & 2 \\
\hline nad6 & - & $\begin{array}{l}13539- \\
14060\end{array}$ & 522 & - & ATG & AGG & 0 \\
\hline $\operatorname{trn} E$ & - & $\begin{array}{l}14061- \\
14128\end{array}$ & 68 & TTC & - & - & 3 \\
\hline$c o b$ & + & $\begin{array}{l}14132- \\
15271\end{array}$ & 1140 & - & ATG & TAG & -1 \\
\hline $\operatorname{trn} T$ & + & $\begin{array}{l}15271- \\
15338\end{array}$ & 68 & TGT & - & - & 1 \\
\hline $\operatorname{trn} P$ & - & $\begin{array}{l}15340- \\
15407\end{array}$ & 68 & TGG & - & - & 0 \\
\hline$C R$ & & $\begin{array}{l}15408- \\
17164\end{array}$ & 1757 & - & - & - & - \\
\hline
\end{tabular}


Table 2: Nucleotide composition of the mitochondrial genomes of different treeshrew mtDNA. The A+T biases of the complete mitogenome, PCGs, tRNAs, rRNA, and CRs were calculated by AT-skew=(A$\mathrm{T}) /(\mathrm{A}+\mathrm{T})$ and $\mathrm{GC}$-skew $=(\mathrm{G}-\mathrm{C}) /(\mathrm{G}+\mathrm{C})$, respectively. 


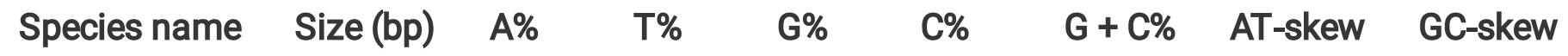

Complete mitogenome

$\begin{array}{lllllllll}\text { T. nicobarica } & 17164 & 32.56 & 25.74 & 14.54 & 27.13 & 41.70 & 0.11 & -0.30 \\ \text { T. belangeri } & 16754 & 32.70 & 26.55 & 14.37 & 26.38 & 40.75 & 0.10 & -0.29 \\ \text { T. minor } & 16187 & 32.06 & 26.83 & 14.77 & 26.32 & 41.09 & 0.08 & -0.28 \\ \text { T. montana } & 16183 & 32.07 & 26.63 & 14.79 & 26.52 & 41.30 & 0.09 & -0.28 \\ \text { T. splendidula } & 16189 & 32.33 & 26.72 & 14.54 & 26.17 & 40.71 & 0.09 & -0.28 \\ \text { T. tana } & 16186 & 32.52 & 27.20 & 14.38 & 25.89 & 40.28 & 0.08 & -0.28\end{array}$

\section{Protein-coding genes (PCGs)}

\begin{tabular}{lllllllll} 
T. nicobarica & 11410 & 31.60 & 26.32 & 13.31 & 28.74 & 42.05 & 0.09 & -0.36 \\
\hline T. belangeri & 11394 & 30.50 & 28.30 & 14.30 & 26.91 & 41.21 & 0.04 & -0.31 \\
\hline T. minor & 11387 & 30.24 & 28.45 & 14.49 & 26.82 & 41.31 & 0.03 & -0.30 \\
\hline T. montana & 11389 & 30.25 & 28.22 & 14.49 & 27.04 & 41.53 & 0.03 & -0.30 \\
\hline T. splendidula & 11362 & 30.50 & 28.37 & 14.33 & 26.81 & 41.14 & 0.04 & -0.30 \\
\hline T. tana & 11389 & 30.89 & 28.72 & 14.00 & 26.39 & 40.39 & 0.04 & -0.31
\end{tabular}

tRNA genes (tRNAs)

\begin{tabular}{|lcccccccc|}
\hline T. nicobarica & 1497 & 34.06 & 26.78 & 17.03 & 22.11 & 39.14 & 0.11 & -0.12 \\
\hline T. belangeri & 1564 & 31.91 & 29.35 & 20.33 & 18.41 & 38.75 & 0.04 & 0.05 \\
\hline T. minor & 1493 & 30.94 & 30.01 & 20.76 & 18.29 & 39.05 & 0.02 & 0.06 \\
\hline T. montana & 1496 & 31.15 & 29.95 & 20.52 & 18.38 & 38.90 & 0.02 & 0.05 \\
\hline T. splendidula & 1496 & 31.75 & 30.21 & 20.12 & 17.91 & 38.03 & 0.02 & 0.06 \\
\hline T. tana & 1497 & 32.00 & 29.86 & 19.97 & 18.17 & 38.14 & 0.03 & 0.05 \\
\hline rRNA genes (rRNAs) & & & & & & & \\
\hline T. nicobarica & 2519 & 35.84 & 22.70 & 18.69 & 22.74 & 41.44 & 0.22 & -0.09 \\
\hline T. belangeri & 2520 & 36.11 & 22.86 & 18.73 & 22.30 & 41.03 & 0.22 & -0.09 \\
\hline T. minor & 2516 & 35.41 & 23.21 & 18.64 & 22.73 & 41.38 & 0.21 & -0.10 \\
\hline T. montana & 2508 & 35.33 & 23.01 & 18.74 & 22.93 & 41.67 & 0.21 & -0.10 \\
\hline T. splendidula & 2514 & 35.52 & 23.31 & 18.70 & 22.47 & 41.17 & 0.21 & -0.09 \\
\hline T. tana & 2510 & 35.54 & 23.86 & 18.53 & 22.07 & 40.60 & 0.20 & -0.09 \\
\hline
\end{tabular}




\begin{tabular}{|c|c|c|c|c|c|c|c|c|}
\hline \multicolumn{9}{|c|}{ Control regions (CRs) } \\
\hline T. nicobarica & 1757 & 33.23 & 25.72 & 14.34 & 26.63 & 40.97 & 0.12 & -0.30 \\
\hline T. belangeri & 1350 & 34.37 & 28.37 & 13.11 & 24.15 & 37.26 & 0.10 & -0.30 \\
\hline T. minor & 788 & 30.20 & 29.95 & 16.50 & 23.35 & 39.85 & 0.00 & -0.17 \\
\hline T. montana & 790 & 30.51 & 29.11 & 16.46 & 23.92 & 40.38 & 0.02 & -0.18 \\
\hline T. splendidula & 778 & 31.75 & 29.18 & 16.32 & 22.75 & 39.07 & 0.04 & -0.16 \\
\hline T. tana & 789 & 30.42 & 28.90 & 16.48 & 24.21 & 40.68 & 0.03 & -0.19 \\
\hline
\end{tabular}

Figures 


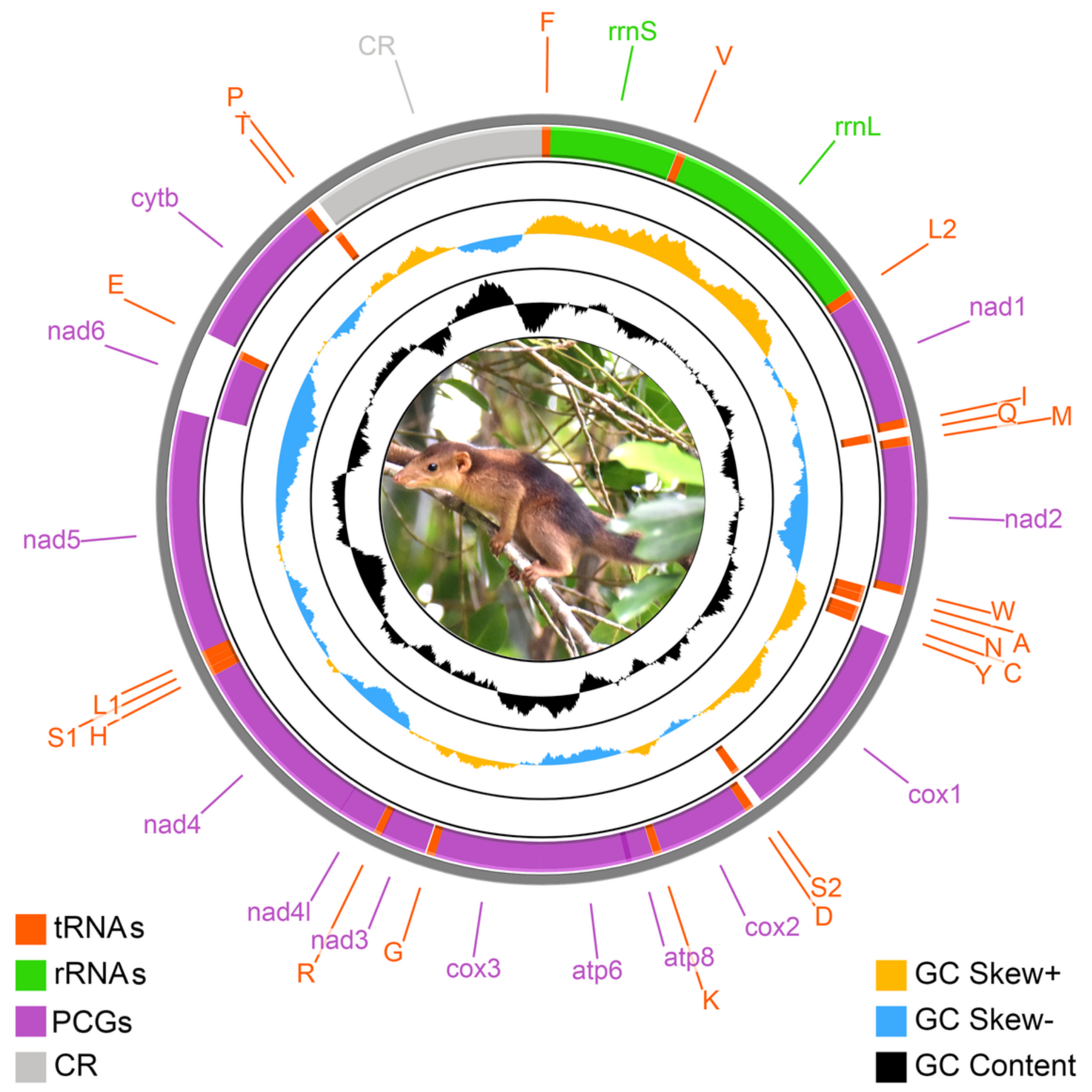

Figure 1

The species photograph and mitochondrial genome of T. nicobarica. Protein-coding genes are marked by orcid color boxes, rRNA genes are marked by green color boxes, tRNA genes are marked by red color boxes, and control region is marked by grey color box. tRNAs are encoded according to their single-letter abbreviations. The GC content is plotted using a black sliding window; GC-skew is plotted using orange and blue color sliding windows as deviation from the average of the complete mitogenome. The figure was illustrated using CGView online server (http://cgview.ca/) with default parameters. The species 
photograph taken by Govindarasu Gokulakrishnan and circular map was merged manually in Adobe Photoshop CS 8.0.

ETAS1

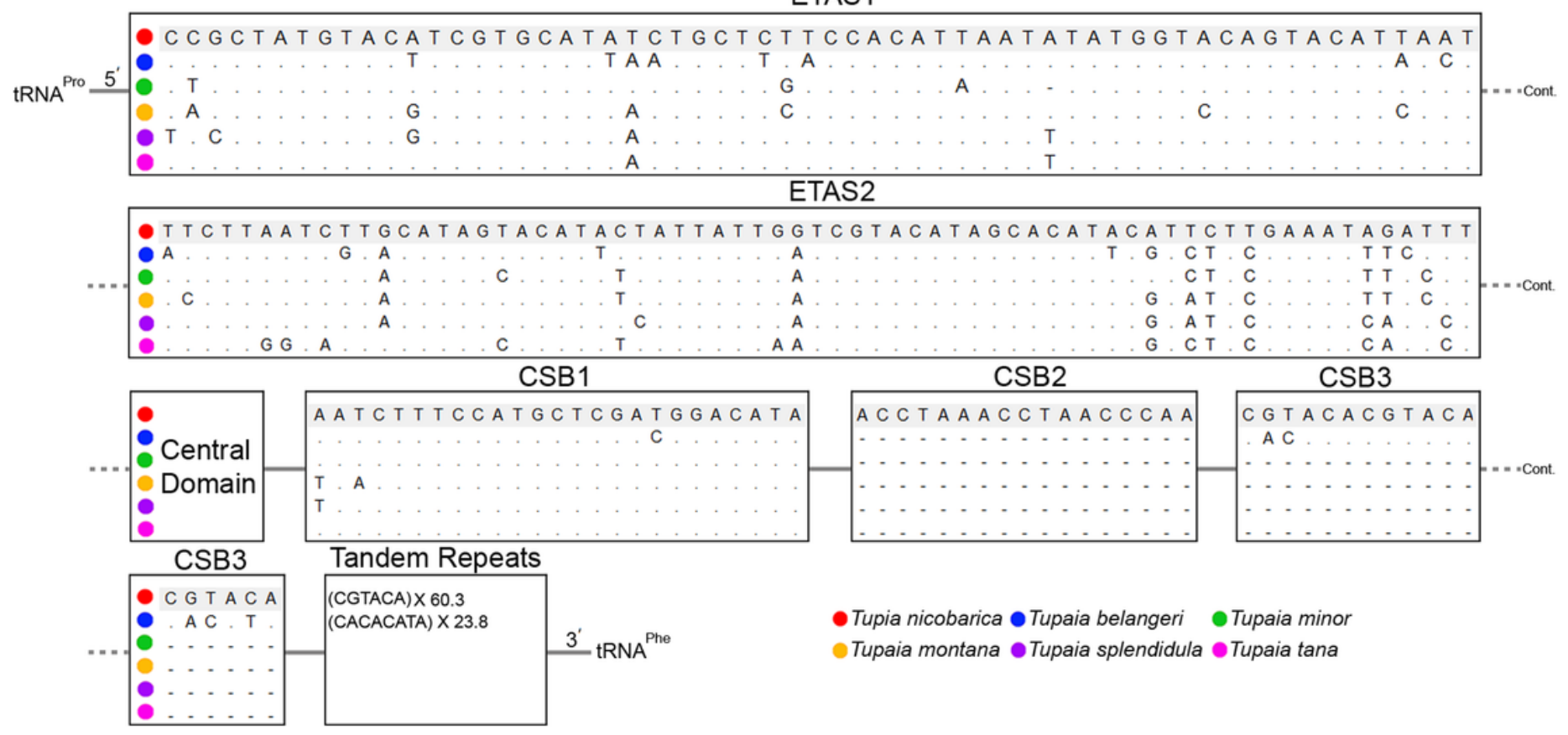

\section{Figure 2}

Comparison of nucleotide composition in different domains of control regions (CRs) and tandem repeats of six Tupaiidae species. The nucleotide compositions were compared through MEGAX software and the tandem repeats were predicted by the online Tandem Repeats Finder web tool (https://tandem.bu.edu/trf/trf.html). The figure was edited manually in Adobe Photoshop CS 8.0.

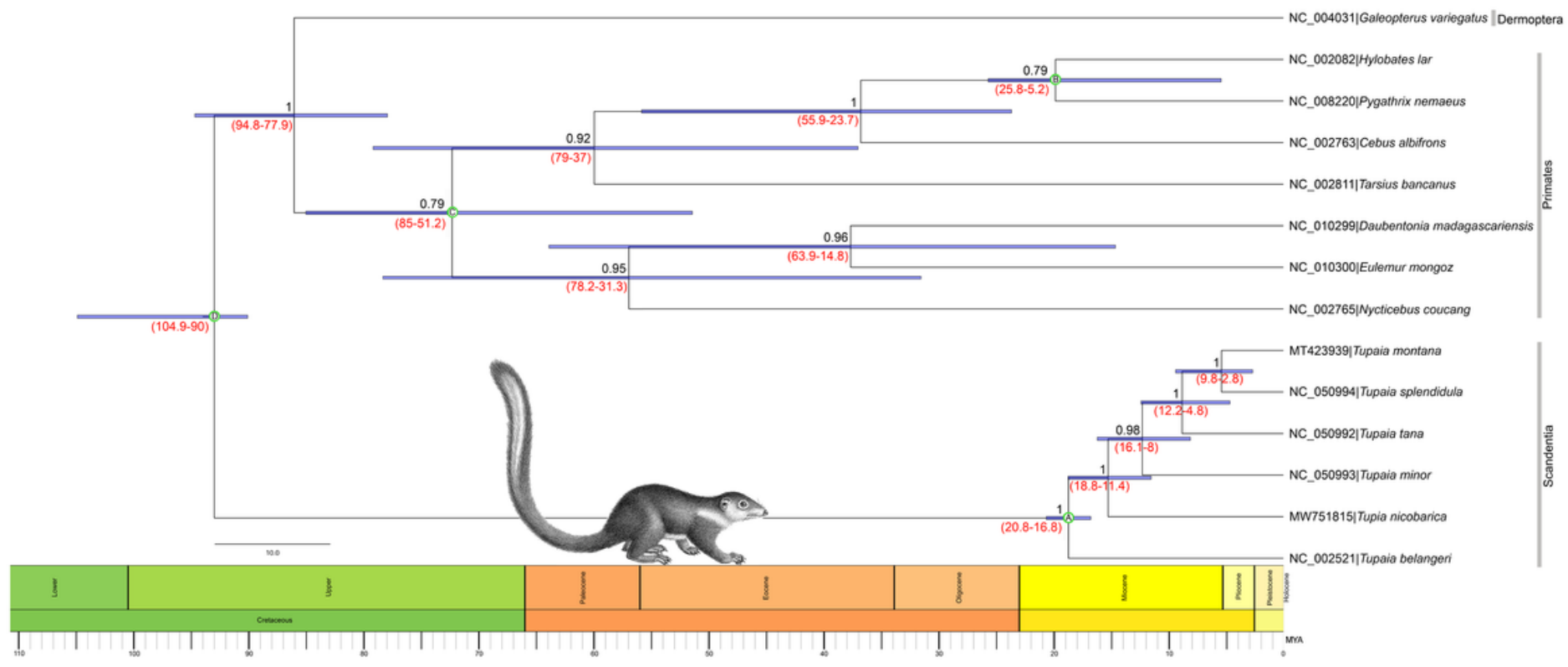

\section{Figure 3}


Bayesian phylogeny showed the molecular timescale for Tupaiidae species evolution compared with other Primates and Dermoptera species. Posterior values of branch lengths were represent by black digit along with each node. The divergence times (in MYR) were estimated by four calibration points (marked by green circle) with GTR+ I+G substitution model and relaxed uncorrelated log-normal clock with the Yule speciation model in BEAST v2.4.7. Blue bars represent $95 \%$ highest probability density (HPD) around mean estimates of divergence times. The range of the estimated divergence times were marked by values in red along with each node. Geological time scale and Tupaiidae species photo (acquired from web) were edited manually in Adobe Photoshop CS 8.0.
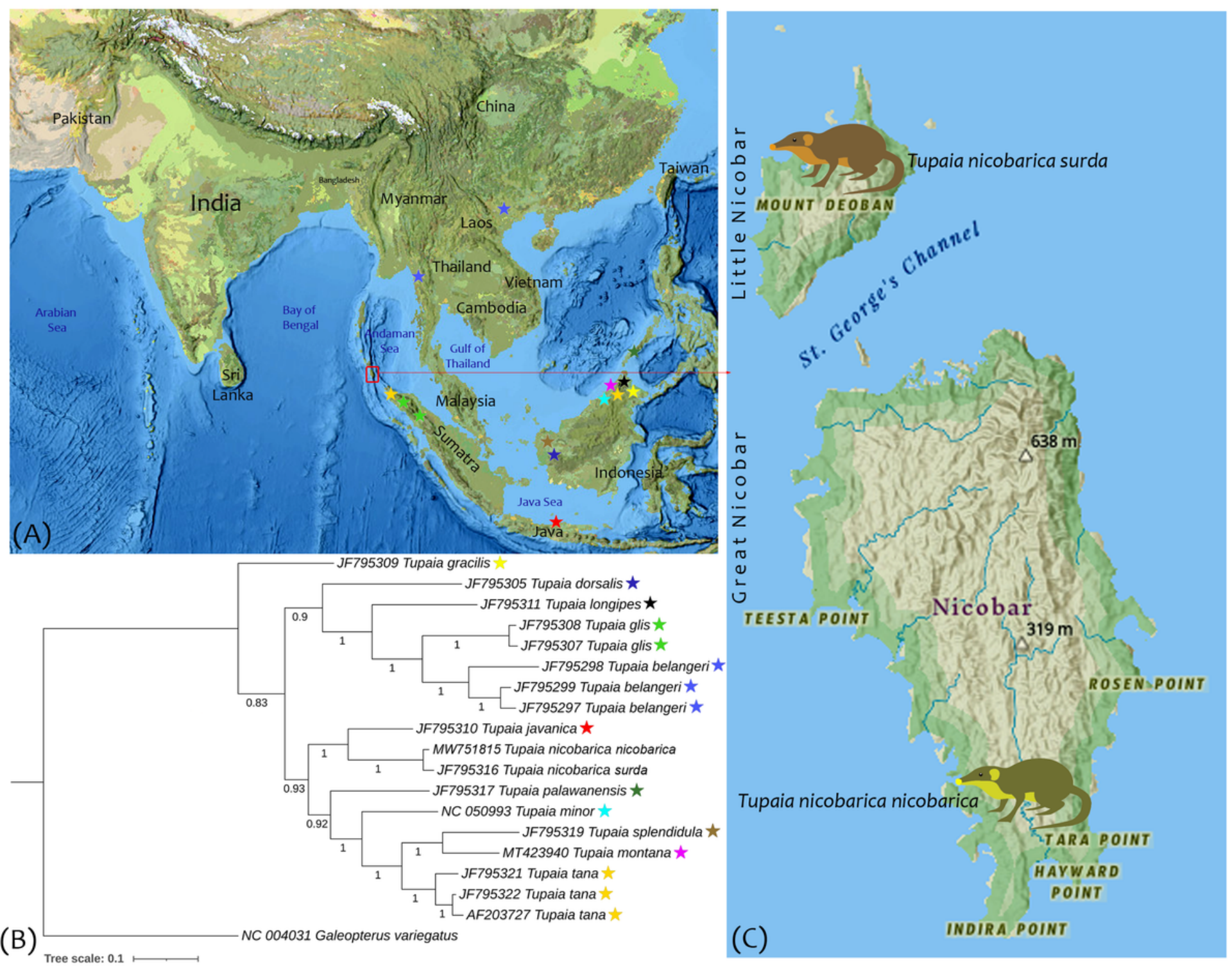

\section{Figure 4}

Genetic status of two known subspecies of T. nicobarica based on 16S rRNA sequences. (A) Map showing the distribution of other comparative Tupaiidae species in the present phylogeny. Map prepared using QGIS 2.6.1 (http://www.qgis.org) and edited manually in Adobe Photoshop CS 8.0. (B) BA Phylogeny showed distinct clustering of T. nicobarica subspecies and other Tupaiidae species. (C) 
Distribution pattern of T. nicobarica nicobarica and T. nicobarica surda in the Great and Little Nicobar Island respectively.

\section{Supplementary Files}

This is a list of supplementary files associated with this preprint. Click to download.

- Supportinginformation.docx 